\title{
Neural Tensor Model for Learning Multi-Aspect Factors in Recommender Systems
}

\author{
Huiyuan Chen and Jing Li \\ Department of Computer and Data Sciences, Case Western Reserve University \\ hxc501@case.edu, jingli@cwru.edu
}

\begin{abstract}
Recommender systems often involve multi-aspect factors. For example, when shopping for shoes online, consumers usually look through their images, ratings, and product's reviews before making their decisions. To learn multi-aspect factors, many context-aware models have been developed based on tensor factorizations. However, existing models assume multilinear structures in the tensor data, thus failing to capture nonlinear feature interactions. To fill this gap, we propose a novel nonlinear tensor machine, which combines deep neural networks and tensor algebra to capture nonlinear interactions among multi-aspect factors. We further consider adversarial learning to assist the training of our model. Extensive experiments demonstrate the effectiveness of the proposed model.
\end{abstract}

\section{Introduction}

Context-aware recommendations have attracted great attention due to their beneficialness of exploring multi-aspect factors [Bhargava et al., 2015; Chen and Li, 2017]. As an example in Figure 1, user reviews are valuable to explain what properties of an item they prefer; Item images provide abundant information, including color, shape, and fashion style. Furthermore, recommending a proper item to users at the right time is also a fundamental task [Karatzoglou et al., 2010]. Incorporating multi-aspect factors is thus a promising strategy to improve recommendation accuracy.

Tensor-based models are naturally preferred due to their ability to express multi-aspect data [Kolda and Bader, 2009]. However, existing tensor models suffer from three drawbacks. First, it is well known that the performance can be hindered by its multilinear assumption, thus failing to capture nonlinear patterns. Second, when the observed tensor is sparse, only mining the tensor provides little information. The coupled tensor-matrix models can alleviate data sparsity by incorporating auxiliary information [Acar et al., 2011; Chen and Li, 2018]. Nevertheless, they need to construct fixed-dimensional feature matrices to couple with tensors. As such, these feature matrices are insufficient to capture the complicated data manifold, especially for image or textual data [Zhang et al., 2017]. Finally, sampling negative training

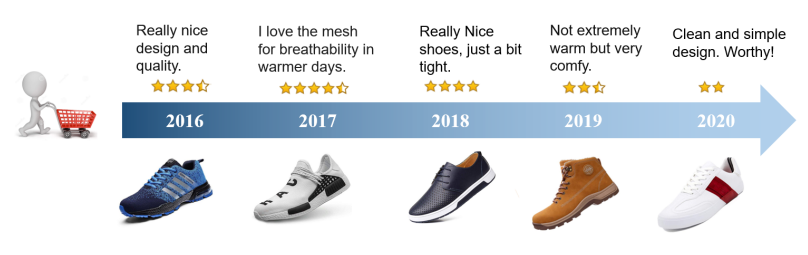

Figure 1: A user's decision involves multi-aspect factors.

instances becomes a nontrivial task since the observed tensor typically only contains positive instances [Yu et al., 2018; Kolda and Bader, 2009; Chen and Li, 2019]. A uniformly random sampling is widely used to generate negative instances. However, those negative samples can be completely unrelated and can be easily discriminated from observed samples, leading to zero loss phenomenon [Wang et al., 2017].

Here we propose a Neural Tensor Machine (NTM), which extends multilinear tensor factorizations by combining the deep neural networks and tensor algebra. Specifically, NTM takes a multi-aspect tensor as input, and aims to learn nonlinear feature interactions among data. NTM contains two components: a shallow GCP layer and a deep tensorized MLP (Figure 2). As such, NTM is able to characterize nonlinear feature interactions. We further consider adversarial learning to supply high-quality negative samples, which improve its generalization performance.

\section{Related Work}

Context-Aware Recommendation. Many studies have focused on using contextual factors to improve system accuracy, such as review-aware [Catherine and Cohen, 2017; Zhang et al., 2017], visually-aware [Yu et al., 2018; He and McAuley, 2016], and time-aware recommendation [Karatzoglou et al., 2010; Wu et al., 2019]. For instance, DeepCoNN [Zheng et al., 2017] and ConvMF [Kim et al., 2016] explored user reviews to study users' behaviors; Vista [He et al., 2016] and DCFA [Yu et al., 2018] incorporated visual features of items to enhance the accuracy; MR [Karatzoglou et al., 2010] and NTF [Wu et al., 2019] exploited temporal behaviors by mining a user-item-time tensor. Inspired by these models, we seek to build a new tensor model that integrate multi-aspect factors together. 
Tensor Factorization. Tensor machines have yielded great promise in context-aware recommendation. Many of them are built upon the multilinear CANDECOMP/PARAFAC (CP) or Tucker tensor factorizations [Kolda and Bader, 2009].

The CP model decomposes a third-order tenor $\mathcal{X} \in$ $\mathbb{R}^{M \times N \times L}$ into three factor matrices: $\mathbf{U} \in \mathbb{R}^{M \times r}, \mathbf{V} \in \mathbb{R}^{N \times r}$, and $\mathbf{W} \in \mathbb{R}^{L \times r}$, such that a tensor entry can be estimated by:

$$
\hat{\mathcal{X}}_{i j k}=\sum_{t=1}^{r} \mathbf{U}_{i t} \mathbf{V}_{j t} \mathbf{W}_{k t}
$$

The Tucker decomposes a tensor $\mathcal{X} \in \mathbb{R}^{M \times N \times L}$ into a core tensor $\mathcal{G} \in \mathbb{R}^{r_{1} \times r_{2} \times r_{3}}$ with three orthogonal factor matrices: $\mathbf{U} \in \mathbb{R}^{M \times r_{1}}, \mathbf{V} \in \mathbb{R}^{N \times r_{2}}$, and $\mathbf{W} \in \mathbb{R}^{L \times r_{3}}$, such that:

$$
\hat{\boldsymbol{\mathcal { X }}}_{i j k}=\sum_{a=1}^{r_{1}} \sum_{b=1}^{r_{2}} \sum_{c=1}^{r_{3}} \mathcal{G}_{a b c} \mathbf{U}_{i a} \mathbf{V}_{j b} \mathbf{W}_{k c}
$$

As we can see, both the CP and Tucker models interpret the three-way interactions using multilinear multiplication. For example, the CP model estimates $\hat{\mathcal{X}}_{i j k}$ by linearly combining of its latent factors with equal contribution. We argue that this strategy may be insufficient to capture nonlinear feature interactions. Our work here builds on this line of work and address this limitation by using deep neural networks.

Nonlinear Tensor Factorization. Recent studies have shown that nonlinear tensor models have superior performance over multilinear tensor models [Fang et al., 2015; Zhe et al., 2016; Xu et al., 2012; Liu et al., 2019; Wu et $a l .$, 2019]. For instance, NLTF [Fang et al., 2015] and InfTucker [Xu et al., 2012] proposed to use Gaussian kernel to capture the nonlinear feature interactions. NTF [Wu et al., 2019] replaced the multilinear operations with multi-layer perceptrons. CoSTCo [Liu et al., 2019] used convolutional neural network to compress the tensor. In contrast, our work explicitly uses tensor algebra to capture triple-wise interactions among multi-aspect factors.

Lastly, it is worth mentioning that our work is different from recent deep tensor networks [Novikov et al., 2015; Socher et al., 2013; Cohen et al., 2016; Lebedev et al., 2015]. The tensor in [Socher et al., 2013] aimed to connect nodes in knowledge graphs. [Novikov et al., 2015; Lebedev et al., 2015; Cohen et al., 2016] focused on establishing relationships between tensor and deep learning, not on nonlinear tensor factorization. Our model here is more general to study nonlinear patterns for multi-aspect tensor data.

\section{Problem Formulation}

\subsection{Problem Setup}

Following [Yu et al., 2018; Karatzoglou et al., 2010], we use a user $\times$ item $\times$ time tensor $\mathcal{X} \in \mathbb{R}^{M \times N \times L}$ to indicate the purchase events, where $M, N$, and $L$ denote the number of users, items, and time intervals, respectively. We consider recommendation problem with implicit feedback: $\boldsymbol{\mathcal { X }}_{i j k}=1$, if user $i$ purchases item $j$ during time interval $k$, otherwise $\boldsymbol{\mathcal { X }}_{i j k}=0$. Our goal is to generate for users a personalized ranking of items that they have not yet provided feedback at certain time [Yu et al., 2018; Wu et al., 2019]. In other words, we aim to infer the unobserved elements $\hat{\boldsymbol{\mathcal { X }}}_{i j k}$ to estimate a score among user $i$, item $j$, and time $k$.

\subsection{Feature Extraction}

In this section, we present the related features in details.

Embedding Look-up. Given a user $i$, an item $j$, and a time interval $k$, their one-hot features $\mathbf{a}_{i} \in \mathbb{R}^{M}, \mathbf{b}_{j} \in \mathbb{R}^{N}$, and $\mathbf{c}_{k} \in \mathbb{R}^{L}$ can be obtained based on their identities. One can obtain dense embeddings via three lookup tables:

$$
\widehat{\mathbf{u}}_{i} \leftarrow \operatorname{lookup}\left(\mathbf{a}_{i}\right), \quad \widehat{\mathbf{v}}_{j} \leftarrow \operatorname{lookup}\left(\mathbf{b}_{i}\right), \quad \widehat{\mathbf{w}}_{k} \leftarrow \operatorname{lookup}\left(\mathbf{c}_{i}\right)
$$

here $\widehat{\mathbf{u}}_{i} \in \mathbb{R}^{d_{1}}, \widehat{\mathbf{v}}_{j} \in \mathbb{R}^{d_{2}}$, and $\widehat{\mathbf{w}}_{k} \in \mathbb{R}^{d_{3}}$ are new embeddings for user $i$, item $j$, and time $k$, respectively.

In addition to identity features, we further extract the features from users' reviews and items' images as follows.

Textual features. We extract features from users' reviews via the KimCNN [Kim, 2014]. Let text $_{i}$ denote the textual reviews of user $i$, which concatenates all reviews written by user $i$ [Zheng et al., 2017], we have:

$$
\mathbf{x}_{i} \leftarrow \operatorname{KimCNN}\left(\text { text }_{i}\right)
$$

here $\mathbf{x}_{i} \in \mathbb{R}^{m}$ is the review-based representation for user $i$.

Visual features. We adopt the well-known Caffe model to extract visual features from images [Jia et al., 2014]. Given an item's image $_{j}$, we obtain its visual feature by pulling out the second fully-connected layer of the Caffe (i.e. FC7):

$$
\mathbf{y}_{j} \leftarrow \mathrm{CNN}\left(\text { image }_{j}\right)
$$

here $\mathbf{y}_{j} \in \mathbb{R}^{n}$ is the image-based representation for item $j$.

Fusion features. We fuse all features together for better representation learning. Given user's features $\left(\widehat{\mathbf{u}}_{i}, \mathbf{x}_{i}\right)$, item's features $\left(\widehat{\mathbf{v}}_{j}, \mathbf{y}_{j}\right)$, and temporal feature $\widehat{\mathbf{w}}_{k}$, we have:

$$
\begin{aligned}
& \mathbf{u}_{i} \leftarrow \operatorname{FC}\left(\Theta_{u} ; \operatorname{CONCAT}\left(\widehat{\mathbf{u}}_{i}, \mathbf{x}_{i}\right)\right), \\
& \mathbf{v}_{j} \leftarrow \operatorname{FC}\left(\Theta_{v} ; \operatorname{ConcAt}\left(\widehat{\mathbf{v}}_{j}, \mathbf{y}_{j}\right)\right), \\
& \mathbf{w}_{k} \leftarrow \widehat{\mathbf{w}}_{k}
\end{aligned}
$$

here $\operatorname{CONCAT}(\cdot)$ is the concatenation; $\left\{\mathbf{u}_{i}, \mathbf{v}_{j}, \mathbf{w}_{k}\right\} \in \mathbb{R}^{r}$ are new representations for user $i$, item $j$, and time $k$, respectively. $\mathbf{w}_{k}$ only contains the one-hot feature since there is no other feature for time. Two fully-connected layer with parameters $\Theta_{u}$ and $\Theta_{v}$ are applied to obtain more sophisticated representations for both users and items. Furthermore, by properly choosing the weights in $\Theta_{u}$ and $\Theta_{v}$, we can reshape the vectors $\mathbf{u}_{i}$ and $\mathbf{v}_{j}$ to have the same dimension as $\mathbf{w}_{k}$. As such, $\left\{\mathbf{u}_{i}, \mathbf{v}_{j}, \mathbf{w}_{k}\right\}$ can be interpreted as latent vectors in CP tensor model as shown latter in Sec. 4.1.

\section{The Proposed Method}

To learn multi-aspect features, a straightforward method is to train a Multi-Layer Perceptron (MLP) [He et al., 2017; Wu et al., 2019] However, MLP fails to capture the triplewise interactions among latent vectors due to its black-box structure, which are important in recommender systems. For example, a user will purchase an item if the item fits the user's preference and if the item is in season (at the right time).

To avoid such information loss, we propose NTM with two modules: a GCP layer and a tensorized MLP layer (Figure 2). 


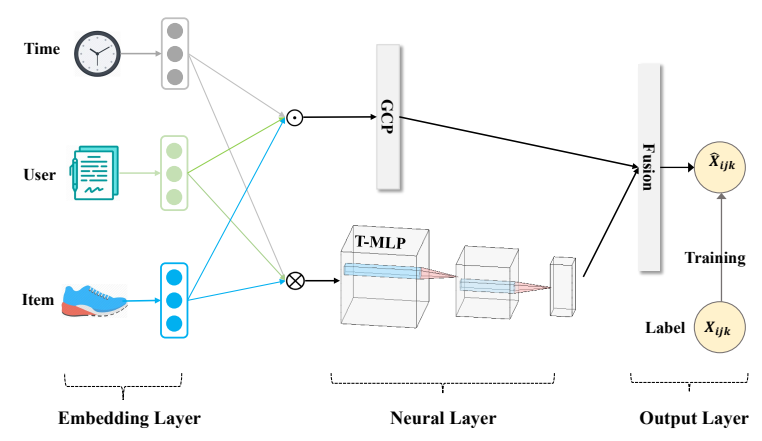

Figure 2: The architecture of the proposed NTM that consists of GCP and T-MLP to exploit the multi-aspect factors.

\subsection{Generalized CP Tensor Factorization}

As shown in Eq. (1), the CP model can interpret triple-wise interactions by its multilinear multiplication. Here we generalize the CP model to learn nonlinear feature interactions.

Given the embeddings $\left\{\mathbf{u}_{i}, \mathbf{v}_{j}, \mathbf{w}_{k}\right\} \in \mathbb{R}^{r}$ from Eq. (4), we design a novel GCP layer $\phi(\cdot)$, which contains a pooling operator that converts a set of embeddings to one vector:

$$
\phi\left(\mathbf{u}_{i}, \mathbf{v}_{j}, \mathbf{w}_{k}\right)=\mathbf{u}_{i} \odot \mathbf{v}_{j} \odot \mathbf{w}_{k}
$$

here $\odot$ is the element-wise product. Clearly, the GCP layer $\phi(\cdot)$ does not introduce extra model parameters, and more importantly, it can be efficiently computed with linear time. Then, we can project the hidden vector $\phi$ into an output layer:

$$
\hat{\mathcal{X}}_{i j k}=a_{\text {out }}\left(\mathbf{h}^{T}\left(\mathbf{u}_{i} \odot \mathbf{v}_{j} \odot \mathbf{w}_{k}\right)\right)
$$

here $a_{\text {out }}$ and $\mathbf{h}$ denote the activation function and weights.

Proposition 1. The CP tensor factorization in Eq. (1) is a special case of generalized tensor factorization in Eq. (6).

Proof. Let $a_{\text {out }}$ be an identity function $\left(a_{\text {out }}(x)=x\right)$, weight $\mathbf{h}$ be a uniform vector of $1\left(\mathbf{h}=[1, \ldots, 1]^{T} \in \mathbb{R}^{r}\right)$, and $\mathbf{u}_{i}(t)$ be the $t$-th element in the column vector $\mathbf{u}_{i}$, we have:

$$
\hat{\mathcal{X}}_{i j k}=a_{\text {out }}\left(\mathbf{h}^{T}\left(\mathbf{u}_{i} \odot \mathbf{v}_{j} \odot \mathbf{w}_{k}\right)\right)=\sum_{t=1}^{r} \mathbf{u}_{i}(t) \mathbf{v}_{j}(t) \mathbf{w}_{k}(t)
$$

which exactly recovers the $\mathrm{CP}$ factorization in Eq. (1) and the embedding size $r$ now becomes the tensor CP rank.

This is a very appealing connection, meaning that we can design a nonlinear CP tensor model. For example, if we adopt a nonlinear function $a_{\text {out }}$ and allow $\mathbf{h}$ to be trained from data without uniform property, GCP provides a more powerful way to express the nonlinear interactions for multi-aspect factors. Here we implement the GCP by using the sigmoid function $\sigma(\cdot)$ and learning $\mathbf{h}$ with the pairwise loss function.

\subsection{Tensorized Multi-layer Perceptron}

To better capture the triple-wise feature interactions, we further propose to use an outer product on $\mathbf{u}_{i}, \mathbf{v}_{j}$, and $\mathbf{w}_{k}$ :

$$
\mathcal{E}=\mathbf{u}_{i} \otimes \mathbf{v}_{j} \otimes \mathbf{w}_{k}
$$

here $\mathcal{E} \in \mathbb{R}^{r \times r \times r}$ is a tensor feature map and $\otimes$ denotes the outer product. Our motivation for $\mathcal{E}$ is straightforward. The $\mathcal{E}$ captures more signals than element-wise product in Eq. (5) since it encodes any tripe-wise interactions. Such strategy is simple but widely used in deep learning [He et al., 2018].

One can flatten $\mathcal{E}$ into a vector and use a MLP to exploit its nonlinearity. However, the size of $\mathcal{E}$ requires massive neurons. Assuming we have a map $\mathcal{E} \in \mathbb{R}^{64 \times 64 \times 64}$ and adopt a MLP with a half-size tower structure. As such, even the first layer requires $262,144 \times 131,072$ parameters in total.

To address this issue, we turn our attention to $n$-mode Product [Kolda and Bader, 2009]: Given a tensor $\mathcal{X} \in \mathbb{R}^{I_{1} \cdots \times I_{n}}$ and a matrix $\mathbf{U} \in \mathbb{R}^{J \times I_{n}}$, the $n$-mode product of $\mathcal{X}$ and $\mathbf{U}$ is denoted as $\mathcal{X} \times{ }_{n} \mathbf{U}$ and is of size $\mathbb{R}^{I_{1} \cdots \times I_{n-1} \times J \times I_{n+1} \cdots \times I_{N}}$. Inspired by this shrinking technique, we propose a Tensorized MLP to successively compress the tensor map $\mathcal{E}$ by using a sequence of three weight matrices along each mode of $\mathcal{E}$ with nonlinear transformation.

T-MLP block: We apply one T-MLP block as:

$$
\mathcal{H}=g\left(\mathcal{E} \times{ }_{1} \mathbf{A} \times{ }_{2} \mathbf{B} \times{ }_{3} \mathbf{C}+\mathcal{B}\right)
$$

here $\mathcal{H}$ is output in the hidden layer; $g(\cdot)$ is the activation function; $\{\mathbf{A}, \mathbf{B}, \mathbf{C}\}$ and $\mathcal{B}$ are the weights and bias. At first glance, our T-MLP is very similar to Tucker in Eq. (2), but they are essentially different. Tucker requires the orthogonality of latent factors and it does not have the bias $\mathcal{B}$ or activation function as in T-MLP.

As such, T-MLP significantly reduces the number of training parameters. For example, we can compress $\mathcal{E}$ with size $\mathbb{R}^{64 \times 64 \times 64}$ to $\mathbb{R}^{16 \times 16 \times 16}$ by weights $\{\mathbf{A}, \mathbf{B}, \mathbf{C}\} \in \mathbb{R}^{16 \times 64}$ and bias $\mathcal{B} \in \mathbb{R}^{16 \times 16 \times 16}$, resulting in $3 \times 16 \times 64+16^{3}$ parameters.

In back propagation, the gradient of weights and bias can be derived using chain rule. We give the gradient of Eq. (8) w.r.t. A inside $g(\cdot)$. Let $\mathcal{J}=\mathcal{E} \times{ }_{1} \mathbf{A} \times{ }_{2} \mathbf{B} \times{ }_{3} \mathbf{C}$ denote the term w.r.t. $\mathbf{A}$, its matricized form is written as: $\boldsymbol{J}_{(1)}=$ $\mathbf{A} \boldsymbol{E}_{(1)}(\mathbf{C} \circ \mathbf{B})^{T}$, where $\circ$ is the Kronecker product; $\boldsymbol{J}_{(1)}$ and $\boldsymbol{E}_{(1)}$ denote the mode-1 matricization of $\mathcal{J}$ and $\mathcal{E}$. We have:

$$
\frac{\partial \boldsymbol{J}_{(1)}}{\partial \mathbf{A}}=\left[(\mathbf{C} \circ \mathbf{B}) \boldsymbol{E}_{(1)}^{T}\right] \circ \mathbf{I}
$$

here $\mathbf{I}$ is the identity matrix. Similar strategy can be used to compute the gradient for $\partial \mathcal{J} / \partial \mathbf{B}, \partial \mathcal{J} / \partial \mathbf{C}$, and $\partial \mathcal{J} / \partial \mathcal{B}$.

Stacking T-MLP blocks: Arguably two of the key contributors of the neural networks are its nonlinearity and the stacking of multiple layers. Likewise, we stack multiple TMLP blocks with $\operatorname{ReLU}(\cdot)$ as the activation function:

$$
\begin{aligned}
& \mathcal{H}_{1}=\operatorname{ReLU}\left(\mathcal{E} \times{ }_{1} \mathbf{A}^{(0)} \times{ }_{2} \mathbf{B}^{(0)} \times{ }_{3} \mathbf{C}^{(0)}+\mathcal{B}^{(0)}\right), \ldots, \\
& \mathcal{H}_{L}=\operatorname{ReLU}\left(\mathcal{H}_{L-1} \times{ }_{1} \mathbf{A}^{(L-1)} \times{ }_{2} \mathbf{B}^{(L-1)} \times{ }_{3} \mathbf{C}^{(L-1)}+\mathcal{B}^{(L-1)}\right),
\end{aligned}
$$

Dropout: Dropout [Srivastava et al., 2014] is a regularization technique for neural networks to prevent overfitting. The idea is simple: randomly "turn off" neurons with probability $\rho$ during training, and use all neurons when testing. To avoid overfitting, we also apply a dropout layer on the feature $\mathcal{E}$, i.e., randomly dropping $\rho$ percent of its elements.

Prediction layer: At last, the output of the last hidden layer $\mathcal{H}_{L}$ is transformed to the final predictive score:

$$
\hat{\mathcal{X}}_{i j k}=\sigma\left(\mathbf{W}_{o} \times \operatorname{Reshape}\left(\mathcal{H}_{L}\right)+\mathbf{b}_{o}\right)
$$

here $\operatorname{Reshape}(\cdot)$ flattens $\mathcal{H}_{L}$ into a vector. The output layer is a fully connected layer with the sigmoid function as predictor. 


\subsection{Joint Learning}

We present our unified model NTM by joint learning GCP and T-MLP. Let $\phi$, and $\mathcal{H}_{L}$ denote the outputs of the last hidden layers of GCP and T-MLP. Then, we merge them as:

$$
\hat{\boldsymbol{X}}_{i j k}=\sigma\left(\mathbf{W}_{f} \times \operatorname{CONCAT}\left(\boldsymbol{\phi}, \operatorname{Reshape}\left(\mathcal{H}_{L}\right)\right)+\mathbf{b}_{f}\right)
$$

this fusion strategy is inspired by the well-known wide\&deep learning [Cheng et al., 2016; He et al., 2017], which has the benefits of memorization and generalization. Our GCP can be regarded as a wide module whereas the T-MLP can be viewed as a deep module. The key difference is that our model aims to learn multi-aspect tensor data, while existing work can only learn two-dimensional matrices.

Pairwise Loss Training. To optimize the model, we opt for the margin-based ranking loss function [Socher et al., 2013]:

$$
\mathcal{L}(\Theta)=\sum_{(i, j, k) \in \mathcal{T}}\left[1+f\left(i, j^{\prime}, k\right)-f(i, j, k)\right]_{+}
$$

where $[x]_{+}=\max (x ; 0) ; f(\cdot)$ and $\Theta$ denote our predictive function and model parameters in Eq. (10); $\mathcal{T}$ denotes the training set, where each instance is a positive triplet i.e., $\boldsymbol{\mathcal { X }}_{i j k}=1 .\left(i, j^{\prime}, k\right)$ is a negative triplet $w . r . t .(i, j, k)$, which can be randomly generated such that $\boldsymbol{\mathcal { X }}_{i j^{\prime} k}=0$.

Although random sampling is commonly used to generate negative triplets, it suffers from zero loss phenomenon [Wang et al., 2018a]. For example, it can easily generate some women's dresses for a male user as negative samples, which contributes little for the model training.

\subsection{Adversarial Training}

To overcome above drawback, we adopt adversarial learning to provide high-quality negative samples in the training step [Wang et al., 2017; Goodfellow et al., 2014]. Concretely, the framework contains a Discriminator D and a Generator $G$. The generator tries to generate useful negative samples, while the discriminator aims to discriminate whether such samples come from $G$ or true data.

Discriminator: The discriminator $D$ aims to minimize:

$$
\begin{aligned}
\mathcal{L}_{D}\left(\Theta_{D}\right)= & \sum_{(i, j, k) \in \mathcal{T}}\left[1+f_{D}\left(i, j^{\prime}, k\right)-f_{D}(i, j, k)\right]_{+} \\
& \left(i, j^{\prime}, k\right) \sim p_{G}\left(i, j^{\prime}, k \mid i, j, k\right)
\end{aligned}
$$

here $p_{G}\left(i, j^{\prime}, k \mid i, j, k\right)$ is the probability for generating $\left(i, j^{\prime}, k\right)$ (as Eq. (14)); $f_{D}$ denotes the loss function, which is identical to $f$ in Eq. (11). The only difference between these two is that Eq. (12) uses negative samples from the generator other than random sampling in Eq. (11).

Generator: The goal of the generator $G$ is to maximize:

$$
\mathcal{L}_{G}\left(\Theta_{G}\right)=\sum_{(i, j, k) \in \mathcal{T}} \mathbb{E}\left[f_{D}\left(i, j^{\prime}, k\right)\right] ; \quad\left(i, j^{\prime}, k\right) \sim p_{G}\left(i, j^{\prime}, k \mid i, j, k\right)
$$

The distribution $p_{G}\left(i, j^{\prime}, k \mid i, j, k\right)$ is:

$p_{G}\left(i, j^{\prime}, k \mid i, j, k\right)=\frac{\exp \left(f_{G}\left(i, j^{\prime}, k\right)\right)}{\sum_{t} \exp \left(f_{G}\left(i, j_{t}^{\prime}, k\right)\right)} ; \quad\left(i, j_{t}^{\prime}, k\right) \in \operatorname{Neg}(i, j, k)$

here $f_{G}$ is the generator function, which is different from the $f_{D}$. Similar to [Wang et al., 2018b], we feed embeddings of

\begin{tabular}{|c|c|c|c|c|}
\hline Dataset & $\mid$ User $\mid$ & $\mid$ Item $\mid$ & $\mid$ Interaction $\mid$ & Density \\
\hline Clothing & 39,371 & 23,022 & 275,547 & $0.031 \%$ \\
Beauty & 22,279 & 12,079 & 192,377 & $0.072 \%$ \\
Cell Phones & 27,872 & 10,361 & 191,648 & $0.067 \%$ \\
Movies \& TV & 101,916 & 47,975 & 984,060 & $0.021 \%$ \\
\hline
\end{tabular}

Table 1: The statistics of Amazon datasets (after 2010)

negative samples into a MLP, $f_{G}:=\operatorname{MLP}\left(\operatorname{concat}\left(\mathbf{u}_{i}, \mathbf{v}_{j^{\prime}}, \mathbf{w}_{k}\right)\right)$. Also, we generate $\operatorname{Neg}(i, j, k)$ by uniformly sampling $N_{r}=$ 60 triplets for each positive triplet, i.e., 60 items that user $i$ has not interacted with at time $k$. We adopt the policy gradient to optimize the generator [Wang et al., 2017]. Its gradient is:

$\nabla \Theta_{G} \mathcal{L}_{G}=\sum_{(i, j, k) \in \mathcal{T}} \mathbb{E}_{\left(i, j^{\prime}, k\right) \sim p_{G}}\left[f_{D}\left(i, j^{\prime}, k\right) \nabla \Theta_{G} \log p_{G}\left(i, j^{\prime}, k \mid i, j, k\right)\right]$

Similar to many GAN-based models, the generator and discriminator are trained alternatively toward their objectives.

\subsection{Computational Complexity}

The computational complexity of our model mainly comes from GCP and T-MLP modules. The complexity for the shallow GCP is $O(r)$, where $r$ is the embedding size in Eq. (4). The complexity for the tensor-matrix multiplication in TMLP is $L \cdot O\left(r^{3} d^{2}\right)$, where $d$ is the embedding size of hidden layers and $L$ is the number of layers. In practice, $L$ and $d$ are typically small and the embedding size $r \ll \min (M, N, L)$ in recommendation problems. The overall complexity can be simplified as $O\left(|c| \cdot r^{3}\right)$, where $|c|$ is a constant.

\section{Experiments}

In this section, we aim to answer the following questions:

RQ1: Do our proposed models capture better nonlinear feature interactions than existing tensor factorizations?

RQ2: Does the proposed NTM outperform the state-of-theart context-aware recommendation methods?

RQ3: What is the influence of various components (e.g., GCP, T-MLP, and sampling strategy) in the NTM?

\subsection{Experimental Setup}

Dataset. We consider four benchmark Amazon ${ }^{1}$ datasets: Clothing Shoes\&Jewelry, Beauty, Cell Phones\&Accessories, and Movies\&TV. The standard 5-core dataset for each category is utilized here. Similar to [Yu et al., 2018], we remove the purchase record before 2010 and discretize the timestamps by weeks, leading to 237 time intervals. The statistics are listed in Table 1. Also, a positive triplet $\mathcal{X}_{i j k}$ means that the user $i$ purchase the item $j$ in the week $k$.

Baselines. (1) CP and Tucker [Kolda and Bader, 2009]: both are linear models. (2) nTucker [Zhe et al., 2016]: a nonlinear Gaussian Tucker. (3) NCF [He et al., 2017]: a neural collaborative filtering model. (4) CoSTCo [Liu et al., 2019]: a CNN-based tensor model. (5) CMTF [Acar et al., 2011]: a coupled tensor-matrix model. (6) VBPR [He and McAuley, 2016]: a Bayesian ranking method. (7) NTF [Wu

\footnotetext{
${ }^{1}$ http://jmcauley.ucsd.edu/data/amazon/index.html
} 
et al., 2019]: a neural model with MLP. (8) DCFA [Yu et al., 2018]: a tensor model with images and time factors. (9) JRL [Zhang et al., 2017]: a model with images and reviews.

Parameter Settings. The parameters for the baselines are initialized as in the original papers and are then carefully tuned to achieve optimal performance. For NTM, its embedding size $r$ in Eq. (4) is searched in [16,32,64,128]. For T-MLP, we employ three hidden layers with dropout ratio 0.5 and each layer sequentially decreases the half size of the input. We implement NTM in PyTorch ${ }^{2}$ with Adam [Kingma and $\mathrm{Ba}, 2015$ ] as optimizer. We use grid-based search to find the best batch size within $[128,256,512,1024]$ and the learning rate within $[0.0005,0.001,0.005,0.01]$.

Evaluation Protocols. We randomly split the datasets into training, validation, and test sets in a 8:1:1 ratio. The validation set is used for tuning hyper-parameters and the final performance is conducted on the test set. We adopt two widely used metrics, Hit@ $k$ and NDCG@ $k$ [He et al., 2017], to evaluate the performance. To avoid heavy computation on all triplets, we follow the strategy in [He et al., 2017; Yu et al., 2018]. For each user $u_{p}$ at time $t_{p}$ in the test set, we randomly sample 100 negative items, and rank these items with the ground-truth items. Based on the ranking results, Hit@ $k$ and NDCG@ $k$ can be evaluated.

\subsection{Effect of Nonlinear Tensor Models (RQ1)}

The proposed models GCP (Eq. (6)), T-MLP (Eq. (9)), and the unified NTM (Eq. (10)) are able to capture nonlinear feature interactions. In this part, we compare them with CP, Tucker, nTucker, NCF, and CoSTCo. These baselines are plain factorization machine without any information of user reviews and item images. For fair comparison, we only use the one-hot features in Eq. (3) and the loss function in Eq. (11) for our methods. We simply omit the Movies\&TV dataset because its large size of data makes Tucker challenging to perform high-order SVD. Due to page limitation, we only show the performance at top-10 scenario, and similar trends can be observed at different top- $k$ scenarios. Table 2 shows the results w.r.t. Hit@10 and NDCG@10.

We can observe that nonlinear tensor models consistently outperform the multilinear CP and Tucker models. nTucker performs better than Tucker due to its nonlinear Gaussian process; GCP outperforms CP since it generalizes CP by using a neural layer. Second, CoSTCo is comparable to nTucker and NCF but worse than GCP. CoSTCo adopts a CNN to perform convolution. Nevertheless, many multi-aspect tensor data may not have spatial locality like images. Third, the performances of T-MLP are better than GCP about $3.1 \%$ on Hit@10 and 3.3\% on NDCG@10, implying the benefit of a deeper tensorized MLP. Finally, NTM achieves the best performance, which demonstrates the high expressiveness of NTM by fusing both wide and deep components.

\subsection{Performance Comparison (RQ2)}

In this section, we compare the overall performance of NTM with the baselines of interest. The results of CP, Tucker, NCF,

\footnotetext{
${ }^{2}$ https://pytorch.org/
}

\begin{tabular}{|c|cc|cc|cc|}
\hline \multirow{2}{*}{ Model } & \multicolumn{2}{|c|}{ Clothing } & \multicolumn{2}{c|}{ Beauty } & \multicolumn{2}{c|}{ Phones } \\
\cline { 2 - 7 } & H@ 10 & N@ 10 & H@ 10 & N@ 10 & H@ 10 & N@ 10 \\
\hline CP & 0.546 & 0.191 & 2.239 & 0.713 & 2.134 & 0.745 \\
Tucker & 0.521 & 0.189 & 2.396 & 0.755 & 2.008 & 0.713 \\
nTucker & 0.816 & 0.283 & 2.607 & 0.909 & 2.544 & 0.902 \\
NCF & 0.965 & 0.407 & 3.012 & 1.135 & 2.598 & 1.178 \\
CoSTCo & 0.983 & 0.426 & 3.187 & 1.189 & 2.632 & 1.235 \\
GCP & 1.272 & 0.466 & 3.225 & 1.285 & 2.866 & 1.329 \\
T-MLP & 1.304 & 0.495 & 3.373 & 1.302 & 2.927 & 1.360 \\
NTM & $\mathbf{1 . 3 5 3}$ & $\mathbf{0 . 5 1 2}$ & $\mathbf{3 . 4 0 1}$ & $\mathbf{1 . 3 2 6}$ & $\mathbf{3 . 0 1 2}$ & $\mathbf{1 . 4 0 4}$ \\
\hline
\end{tabular}

Table 2: Results of pure tensor models. (all number are percentage numbers without $\%, \mathrm{H}$ is short for Hit and $\mathrm{N}$ is for NDCG).

CoSTCo, and nTucker are omitted due to their inferior performances without auxiliary data. Figure 3 shows the top- $k$ performance, where $k$ is set to 5,10 , and 15 .

As shown in Figure 3, we observe that deep neural models (e.g., NTF, DCFA, JRL, and NTM) substantially outperform the shallow methods (e.g, CMTF and VBPR), indicating the superiority of deep neural networks. More importantly, unlike requiring pre-defined feature matrices in the CMTF, these models allow end-to-end learning for contextual factors like user reviews and item images without manually crafted combinatorial features. Moreover, NTF performs much better than VBPR and CMTF, but falls behind DCFA, JRL, and NTM. This is because NTF only relies on the identity features of users and items. In contrast, DCFA alleviates this issue by incorporating visual features from item images. JRL and NTM further improve the quality of recommendations by joint exploring the contextual factors of user reviews and item images. Finally, NTM achieves the best performance over all the comparison methods, showing an average improvement of $24.6 \%$ and $25.7 \%$ than the state-of-the-art JRL model in terms of Hit@ $k$ and NDCG@ $k$, respectively. The improvements of NTM mainly come from its wide and deep components for modeling triple-wise nonlinear interactions of multiaspect factors. In addition, NTM adopts the effectiveness of a self-adversarial negative sampling technique to assist the model training (see Figure 5(b)).

\subsection{Study of NTM (RQ3)}

Ablation Study. We analyze the influence of each component via ablation studies. For each variant, we simply remove one module and compare the results with the default NTM.

Table 3 shows the performance of five variants on Clothing and Beauty datasets. Our results are summarized as follows: 1) Remove GAN: we find that our GAN-based sampling method can provide high-quality negative samples for NTM, resulting in a better performance. In contrast, randomly sampling negative samples may be effortlessly discriminated in the training step; 2) Remove GCP: the performances are inferior without GCP layer. Presumably this is because our wide component GCP serve an important role of generalization in the Wide\&Deep learning; 3) Remove T-MLP: this variant substantially decreases the overall performance with a large margin, verifying the effectiveness of the T-MLP in capturing useful interactions from feature outer product space; 4) Remove image CNN or Review $C N N$ : not surprisingly, deleting the module of either user reviews or item images significantly 

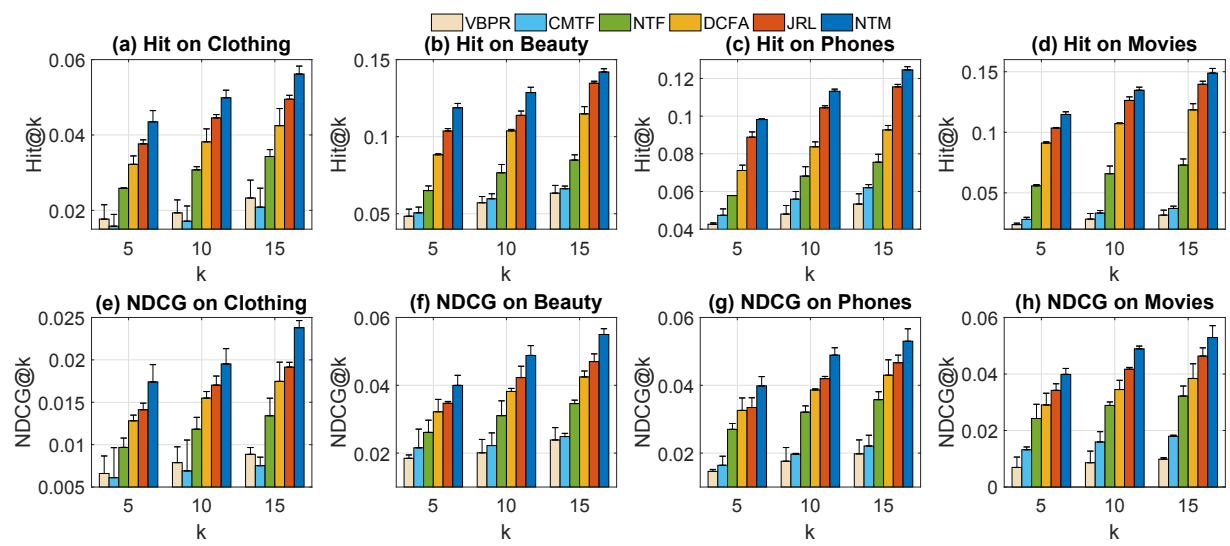

Figure 3: Performance of Top- $k$ recommendations in terms of Hit@ $k$ (3a-3d) and NDCG@ $k$ (3e-3h) with error bars.

\begin{tabular}{|c|cc|cc|}
\hline \multirow{2}{*}{ Ablation Study } & \multicolumn{2}{|c|}{ Clothing } & \multicolumn{2}{c|}{ Beauty } \\
\cline { 2 - 5 } & Hit@ 10 & NDCG@ 10 & Hit@ 10 & NDCG@ 10 \\
\hline NTM & 4.992 & 1.986 & 12.931 & 4.902 \\
Remove GAN & 4.670 & 1.803 & 12.113 & 4.712 \\
Remove GCP & 4.691 & 1.831 & 12.275 & 4.763 \\
Remove T-MLP & $4.023 \downarrow$ & $1.691 \downarrow$ & $10.615 \downarrow$ & $4.124 \downarrow$ \\
Remove Image CNN & $3.881 \downarrow$ & $1.563 \downarrow$ & $10.218 \downarrow$ & $3.853 \downarrow$ \\
Remove Review CNN & $3.782 \downarrow$ & $1.547 \downarrow$ & $10.324 \downarrow$ & $3.902 \downarrow$ \\
\hline
\end{tabular}

Table 3: Ablation analysis on our model (all numbers are percentage without $\%, ' \downarrow$ ' means a severe performance drop).

hurts the overall performance. This implies that both reviewbased CNN features and image-based CNN features are important to understand users' preference.

Embedding Size of NTM. The embedding size $r$ in Eq. (4) is a key hyper-parameter since it affects the representation ability of NTM. Here we vary embedding size $r$ within $[16,32,64,128]$ and compare with the JRL model in terms of Hit@10 and NDCG@10 on Clothing dataset. As shown in Figure 4(a)-4(b), we observe that both NTM and JRL models benefit from a large embedding size. In general, NTM achieves satisfactory performance with $r \geq 64$. Assessments on other datasets are similar and omitted here.

Layers of NTM. We also conduct experiments to see whether using a deeper network architecture is beneficial to the recommendation task. To this end, we vary the number of T-MLP blocks in the NTM within $L=[2,3,4,5]$. As shown
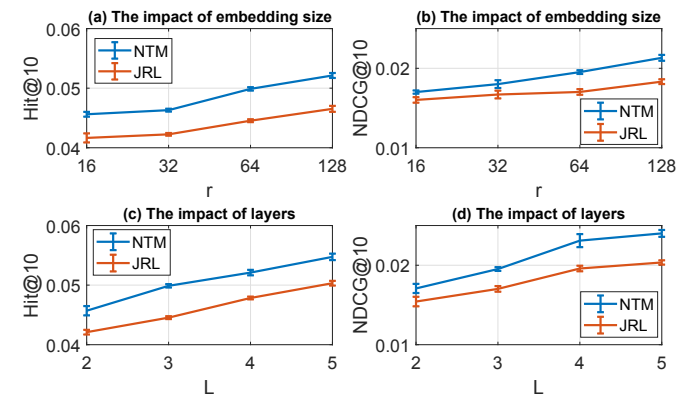

Figure 4: The impact of embedding sizes $r(4 a-4 b)$, and number of layers $L(4 \mathrm{c}-4 \mathrm{~d})$. on Clothing dataset.
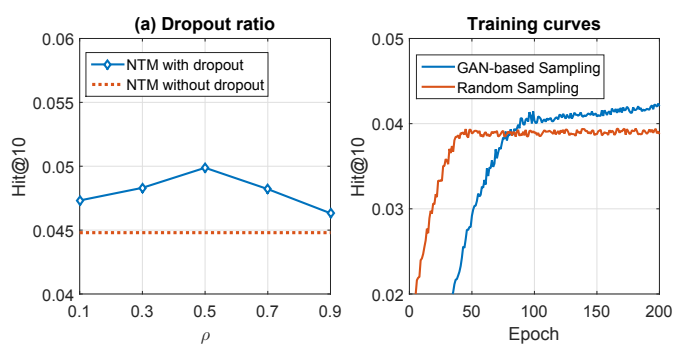

Figure 5: (a) The impact of dropout ratio. (b) Training loss w.r.t Eq. (11) and Eq. (12). Both are for Clothing datasets.

in Figure 4(c)-4(d), stacking more layers gradually enhances the recommendation performance. We attribute the improvements to the usage of stacking more nonlinear layers to model complex user-item-time interactions.

Dropout and Adversarial Training. Figure 5(a) shows the performances of NTM w.r.t. dropout ratio. Our results show that dropout offers better performance. Specifically, using a dropout ratio $\rho \approx 0.5$ achieves an optimal accuracy.

The training progress w.r.t. Eq. (11) and Eq. (12) are also shown in Figure 5(b). The performance of our GANbased sampling method is always in increasing trends, verifying the effectiveness of its high-quality negative training samples. In contrast, random negative samples are often too trivial to fit the model, which possibly leads to zero loss phenomenon [Wang et al., 2018a].

\section{Conclusion}

In this work, we propose a nonlinear tensor machine that combines deep neural networks and tensor algebra to investigate the impacts of multi-aspect factors. We also develop a self-adaptive negative sampling strategy to assist the model training. Experimental results demonstrate the effectiveness of our proposed model for context-aware recommendations. For future work, we will incorporate more contextual factors such as location and user social network.

\section{Acknowledgments}

This work has been supported in part by NSF CCF1815139. 


\section{References}

[Acar et al., 2011] Evrim Acar, Tamara G Kolda, and Daniel M Dunlavy. All-at-once optimization for coupled matrix and tensor factorizations. Computing Research Repository (CORR), 2011.

[Bhargava et al., 2015] Preeti Bhargava, Thomas Phan, Jiayu Zhou, and Juhan Lee. Who, what, when, and where: Multi-dimensional collaborative recommendations using tensor factorization on sparse user-generated data. In $W W W, 2015$.

[Catherine and Cohen, 2017] Rose Catherine and William Cohen. Transnets: Learning to transform for recommendation. In RecSys, 2017.

[Chen and Li, 2017] Huiyuan Chen and Jing Li. Learning multiple similarities of users and items in recommender systems. In ICDM, 2017.

[Chen and Li, 2018] Huiyuan Chen and Jing Li. Drugcom: Synergistic discovery of drug combinations using tensor decomposition. In ICDM, 2018.

[Chen and Li, 2019] Huiyuan Chen and Jing Li. Adversarial tensor factorization for context-aware recommendation. In RecSys, 2019.

[Cheng et al., 2016] Heng-Tze Cheng, Levent Koc, Jeremiah Harmsen, Tal Shaked, Tushar Chandra, Hrishi Aradhye, Glen Anderson, Greg Corrado, Wei Chai, Mustafa Ispir, et al. Wide \& deep learning for recommender systems. In DLRS, 2016.

[Cohen et al., 2016] Nadav Cohen, Or Sharir, and Amnon Shashua. On the expressive power of deep learning: A tensor analysis. In COLT, 2016.

[Fang et al., 2015] Xiaomin Fang, Rong Pan, Guoxiang Cao, Xiuqiang He, and Wenyuan Dai. Personalized tag recommendation through nonlinear tensor factorization using gaussian kernel. In AAAI, 2015.

[Goodfellow et al., 2014] Ian Goodfellow, Jean Pouget-Abadie, Mehdi Mirza, Bing Xu, David Warde-Farley, Sherjil Ozair, Aaron Courville, and Yoshua Bengio. Generative adversarial nets. In NeurIPS, 2014.

[He and McAuley, 2016] Ruining He and Julian McAuley. Vbpr: visual bayesian personalized ranking from implicit feedback. In AAAI, 2016.

[He et al., 2016] Ruining He, Chen Fang, Zhaowen Wang, and Julian McAuley. Vista: A visually, socially, and temporally-aware model for artistic recommendation. In RecSys, 2016.

[He et al., 2017] Xiangnan He, Lizi Liao, Hanwang Zhang, Liqiang $\mathrm{Nie}, \mathrm{Xia} \mathrm{Hu}$, and Tat-Seng Chua. Neural collaborative filtering. In $W W W, 2017$.

[He et al., 2018] Xiangnan He, Xiaoyu Du, Xiang Wang, Feng Tian, Jinhui Tang, and Tat-Seng Chua. Outer product-based neural collaborative filtering. In IJCAI, 2018.

[Jia et al., 2014] Yangqing Jia, Evan Shelhamer, Jeff Donahue, Sergey Karayev, Jonathan Long, Ross Girshick, Sergio Guadarrama, and Trevor Darrell. Caffe: Convolutional architecture for fast feature embedding. In $M M, 2014$.

[Karatzoglou et al., 2010] Alexandros Karatzoglou, Xavier Amatriain, Linas Baltrunas, and Nuria Oliver. Multiverse recommendation: n-dimensional tensor factorization for context-aware collaborative filtering. In RecSys, 2010.

[Kim et al., 2016] Donghyun Kim, Chanyoung Park, Jinoh Oh, Sungyoung Lee, and Hwanjo Yu. Convolutional matrix factorization for document context-aware recommendation. In RecSys, 2016.
[Kim, 2014] Yoon Kim. Convolutional neural networks for sentence classification. In EMNLP, 2014.

[Kingma and Ba, 2015] Diederik P Kingma and Jimmy Ba. Adam: A method for stochastic optimization. In ICLR, 2015.

[Kolda and Bader, 2009] Tamara G Kolda and Brett W Bader. Tensor decompositions and applications. SIAM review, 51(3):455500, 2009.

[Lebedev et al., 2015] Vadim Lebedev, Yaroslav Ganin, Maksim Rakhuba, Ivan Oseledets, and Victor Lempitsky. Speeding-up convolutional neural networks using fine-tuned cp-decomposition. In ICLR, 2015.

[Liu et al., 2019] Hanpeng Liu, Yaguang Li, Michael Tsang, and Yan Liu. Costco: A neural tensor completion model for sparse tensors. In $K D D, 2019$.

[Novikov et al., 2015] Alexander Novikov, Dmitrii Podoprikhin, Anton Osokin, and Dmitry P Vetrov. Tensorizing neural networks. In NeurIPS, 2015.

[Socher et al., 2013] Richard Socher, Danqi Chen, Christopher D Manning, and Andrew Ng. Reasoning with neural tensor networks for knowledge base completion. In NeurIPS, 2013.

[Srivastava et al., 2014] Nitish Srivastava, Geoffrey Hinton, Alex Krizhevsky, Ilya Sutskever, and Ruslan Salakhutdinov. Dropout: a simple way to prevent neural networks from overfitting. The journal of machine learning research, 15(1):1929-1958, 2014.

[Wang et al., 2017] Jun Wang, Lantao Yu, Weinan Zhang, Yu Gong, Yinghui Xu, Benyou Wang, Peng Zhang, and Dell Zhang. Irgan: A minimax game for unifying generative and discriminative information retrieval models. In SIGIR, 2017.

[Wang et al., 2018a] Peifeng Wang, Shuangyin Li, and Rong Pan. Incorporating gan for negative sampling in knowledge representation learning. In $A A A I, 2018$.

[Wang et al., 2018b] Qinyong Wang, Hongzhi Yin, Zhiting Hu, Defu Lian, Hao Wang, and Zi Huang. Neural memory streaming recommender networks with adversarial training. In $K D D$, 2018.

[Wu et al., 2019] Xian Wu, Baoxu Shi, Yuxiao Dong, Chao Huang, and Nitesh V Chawla. Neural tensor factorization for temporal interaction learning. In WSDM, 2019.

[Xu et al., 2012] Zenglin Xu, Feng Yan, and Yuan Qi. Infinite tucker decomposition: Nonparametric bayesian models for multiway data analysis. In ICML, 2012.

[Yu et al., 2018] Wenhui Yu, Huidi Zhang, Xiangnan He, Xu Chen, Li Xiong, and Zheng Qin. Aesthetic-based clothing recommendation. In $W W W, 2018$.

[Zhang et al., 2017] Yongfeng Zhang, Qingyao Ai, Xu Chen, and W Bruce Croft. Joint representation learning for top-n recommendation with heterogeneous information sources. In $C I K M$, 2017.

[Zhe et al., 2016] Shandian Zhe, Kai Zhang, Pengyuan Wang, Kuang-chih Lee, Zenglin Xu, Yuan Qi, and Zoubin Ghahramani. Distributed flexible nonlinear tensor factorization. In NeurIPS 2016.

[Zheng et al., 2017] Lei Zheng, Vahid Noroozi, and Philip S Yu. Joint deep modeling of users and items using reviews for recommendation. In WSDM, 2017. 